\title{
Monocyte-to-high-density lipoprotein cholesterol ratio is associated with the presence and size of thyroid nodule irrespective of the gender
}

\author{
Xing Zhen $\mathrm{Liu}^{1 \dagger} \mathrm{D}$, Jun Min Wang ${ }^{1 \dagger}$, Yi Xin $\mathrm{Ji}^{i^{*}}$ and Dong Bao Zhao ${ }^{2^{*}}$
}

\begin{abstract}
Background: Systemic inflammation may be involved in the formation and progression of thyroid nodule (TN). The aim of this large-scale study was to investigate the association of several simple inflammatory markers with the presence and size of $\mathrm{TN}$.

Methods: A total of 133,698 adults were included for the current analysis. The neutrophil-to-lymphocyte ratio (NLR), platelet-to-lymphocyte ratio (PLR), lymphocyte-to-monocyte ratio (LMR), and monocyte-to-high-density lipoprotein cholesterol ratio (MHR) were calculated. The logistic regression was used to explore the association of the four markers with the presence and size of TN.

Results: The prevalence of TN was $55.1 \%$ among females and $44 \%$ among males; $13 \%$ of women and $8 \%$ of men had non-micronodule. In women, MHR and PLR were significantly associated with the presence of TN and nonmicronodule; in men, MHR and NLR were significantly associated with the presence of TN and non-micronodule.

Conclusions: As a low-cost, simple, and reproducible inflammatory marker, MHR is strongly associated with the presence and size of TN irrespective of the gender.
\end{abstract}

Keywords: Thyroid nodule, Inflammatory markers, Biomarkers

\section{Introduction}

Thyroid nodule (TN) are extremely common disorder of the endocrine system, which present in more than $50 \%$ of the randomly selected population [1]. Although most TN are benign, about $7-15 \%$ are malignant and $5 \%$ causing symptoms or thyroid dysfunction [2]. Some well-known factors involved in the formation and progression of $\mathrm{TN}$, such as age, gender, iodine deficiency, and thyroid-stimulating hormone (TSH) [3]. More recently, the role of systemic inflammatory response caused by unhealthy lifestyle such as high calorie intake, sedentary behaviour, and chronic stress in the

\footnotetext{
* Correspondence: jiyixinbj@sohu.com; dongbaozhao@163.com

${ }^{+}$Xing Zhen Liu and Jun Min Wang contributed equally to this work.

'Hangzhou Aeronautical Sanatorium of Chinese Air Force, Hangzhou, No. 27, Yang Gong Di, Xihu District, Zhejiang 310007, Hangzhou, China

2Department of Rheumatology, Changhai Hospital, Naval Military Medical University (The Second Military Medical University), No. 168 Changhai Road, Yangpu District, Shanghai 200433, China
}

pathophysiology of TN has also attracted the interest of researchers [4-6].

In clinical practice, C-reactive protein (CRP) and erythrocyte sedimentation rate (ESR) are well established markers for detection the inflammatory conditions [7]. Nevertheless, these traditional inflammatory markers are not widely used in primary care due to their cost, difficulty in interpretating abnormal results, and possible cascade of further tests [8]. Therefore, several simple and cost-effective inflammatory markers, such as neutrophil-to-lymphocyte ratio (NLR), platelet-tolymphocyte ratio(PLR), lymphocyte-to-monocyte ratio (LMR), and monocyte-to-high-density lipoprotein cholesterol ratio (MHR), have been developed and established links with some tumors and cardiovascular disease [9-11].

Currently, there are no data on the relationship between aforementioned inflammatory indicators and $\mathrm{TN}$. 
In order to address these research gaps, this large-scale cross section study was conducted to explore the possible association of the four simple and cost-effective inflammatory markers with the presence and size of TN.

\section{Methods}

\section{Study population}

This cross-sectional study was based on the database of subjects who received routine physical examination between November 2015 and January 2019 in China's Yangtze River Delta region, where is not obvious iodine deficiency area. Subjects with history of other thyroid disease and connective tissue diseases, and those taking amiodarone, glucocorticoid, and estrogens were excluded. Then a total of 133,698 adults with complete data were included in the final analysis. The ethics committee of Hangzhou Aeronautical Sanatorium of Chinese Air Force approved the study protocol.

\section{Data collection}

Basic personal and family medical histories and medication use were collected. Anthropometric indicators [height, weight, waist circumference (WC), and hip circumference $(\mathrm{HC})$ ] were measured by well-trained examiners. Systolic blood pressures (SBP) and diastolic blood pressures (DBP) were obtained 3 times on the right arm by trained technicians using a standardized automatic electronic sphygmomanometer. The blood specimens of subjects were collected after a minimum of $8 \mathrm{~h}$ of overnight fasting. The peripheral blood cells (e.g., white blood cell, lymphocytes, neutrophils, monocytes, eosinophils, basophils, and platelets) were analyzed using automated hematology system (Beckman Coulter, Inc., USA). (reviewer \#1, comment \#1) The fasting plasma glucose (FPG) and serum lipid profle [total cholesterol (TC), triglycerides (TG), high-density lipoprotein cholesterol (HDLc), and low-density lipoprotein cholesterol (LDLc)] were assayed using an Automatic Biochemistry Analyzer (HITACHI, Inc., Japan). (reviewer \#1, comment \#2).

All thyroid ultrasonographic examinations were performed by qualified radiologists using a $5-12 \mathrm{MHz}$ linear probe according to a standard procedure, and carried out on the individuals lying on examination bed with the neck extended. A TN was defned as discrete lesions within the thyroid gland distinct from the surrounding thyroid parenchyma, and which had a solid portion regardless of the presence of a cystic portion. A maximum diameter of $\geq 3 \mathrm{~mm}$ was used to identify the presence of $\mathrm{TN}$ and the maximum diameter for $\mathrm{TN}>10 \mathrm{~mm}$ was defined as thyroid non-micronodule.

\section{Definitions}

BMI was calculated as weight divided by the square of height, and WC divided by the HP was waist to hip ratio
(WHR). The calculation of NLR, PLR, and LMR were the division of the corresponding absolute count of blood cells, and MHR was calculated as the ratio of the absolute monocyte count divided by the HDLc.

\section{Statistical analysis}

Statistical analysis was performed using SPSS 18.0 (SPSS Inc.). Data are expressed as numbers (percentage) or means \pm SD. Categorical variables were analyzed using the chi-squared test, and independent sample t-test were used to compare the differences of continuous variables. Logistic regression analyses was applied to explore the associations of the four inflammatory markers with the presence and size of TN. NLR, PLR, LMR, and MHR were divided into four quartiles, and the lowest quartile was used as a reference (the highest quartile of LMR was used as a reference). The age, genders, obesity, hypertension, and diabetes were adjusted. $P$-value $<0.05$ was considered statistically signifcant.

\section{Results}

The general characteristics of individuals are summarized in Table 1 . The total of 133,698 subjects included $37.9 \%$ women and $62.1 \%$ men. The mean age was $46.5 \pm$ 12.9 years and the prevalence of TN was $48.2 \%$. The TN group had higher age, anthropometric indicators, BP,

Table 1 General characteristics in the thyroid nodule group and the non-thyroid nodule group

\begin{tabular}{llll}
\hline Parameters & Non-TN & TN & $P$ value \\
\hline No., $\mathrm{n}(\%)$ & $69,232(51.8)$ & $64,466(48.2)$ & $<0.001$ \\
Age, $y$ & $42.8 \pm 11.4$ & $50.4 \pm 13.1$ & $<0.001$ \\
BMI $\left(\mathrm{kg} / \mathrm{m}^{2}\right)$ & $23.6 \pm 3.3$ & $23.9 \pm 3.2$ & $<0.001$ \\
WC $(\mathrm{cm})$ & $79.8 \pm 10.0$ & $80.6 \pm 9.9$ & $<0.001$ \\
WHR & $0.84 \pm 0.07$ & $0.85 \pm 0.07$ & $<0.001$ \\
SBP $(\mathrm{mmHg})$ & $122.3 \pm 16.5$ & $125.7 \pm 18.1$ & $<0.001$ \\
DBP $(\mathrm{mm} \mathrm{Hg})$ & $75.7 \pm 11.5$ & $76.7 \pm 11.7$ & $<0.001$ \\
FPG $(\mathrm{mmol} / \mathrm{L})$ & $5.65 \pm 1.09$ & $5.88 \pm 1.33$ & $<0.001$ \\
TC $(\mathrm{mmol} / \mathrm{L})$ & $4.76 \pm 0.89$ & $4.85 \pm 0.92$ & $<0.001$ \\
TG $(\mathrm{mmol} / \mathrm{L})$ & $1.54 \pm 1.30$ & $1.57 \pm 1.27$ & $<0.001$ \\
LDLc $(\mathrm{mmol} / \mathrm{L})$ & $2.56 \pm 0.74$ & $2.61 \pm 0.75$ & $<0.001$ \\
HDLc $(\mathrm{mmol} / \mathrm{L})$ & $1.48 \pm 0.34$ & $1.49 \pm 0.35$ & $<0.001$ \\
WBC $\left(\times 10^{9} / \mathrm{L}\right)$ & $6.39 \pm 1.55$ & $6.36 \pm 1.60$ & $<0.001$ \\
Platelet $\left(\times 10^{9} / \mathrm{L}\right)$ & $204.9 \pm 49.8$ & $203.9 \pm 51.1$ & $<0.001$ \\
Neutrophil $\left(\times 10^{9} / \mathrm{L}\right)$ & $3.68 \pm 1.19$ & $3.69 \pm 1.22$ & 0.250 \\
Lymphocyte $\left(\times 10^{9} / \mathrm{L}\right)$ & $2.12 \pm 0.58$ & $2.09 \pm 0.60$ & $<0.001$ \\
Monocyte $\left(\times 10^{9} / \mathrm{L}\right)$ & $0.39 \pm 0.13$ & $0.38 \pm 0.12$ & $<0.001$ \\
\hline TN $)$ & &
\end{tabular}

TN Thyroid nodules, BMI Body mass index, WC Waist circumference, WHR Waist to hip ratio, SBP Systolic blood pressure, DBP Diastolic blood pressure, FPG Fasting plasma glucose, TC Total cholesterol, TG Triglyceride, HDLC Highdensity lipoprotein cholesterol, $L D L c$ Low-density lipoprotein cholesterol, WBC White blood cell 
FPG, TC, TG, LDLc, HDLc, and neutrophil count, but lower white blood cell, platelets, lymphocyte, and monocyte than that of non-TN group. (reviewer \#1, comment \#3).

After grouping by gender, the prevalence of TN was $55.1 \%$ among females and $44 \%$ among males; $13 \%$ of women and $8 \%$ of men had non-micronodule. The mean values of the four inflammatory markers according to the presence and size of thyroid nodule are presented in Table 2. Among women, the TN group had higher LMR and lower NLR than that of non-TN group, no significant difference in PLR and MHR between the two groups; the non-micronodule group had higher MHR than that of micronodule group, no significant differences in other inflammation indicators between the two groups. Among men, the TN and non-micronodule group all had higher NLR and MHR, but lower LMR than that of non-TN and micronodule group. (reviewer \#1, comment \#4) Among women, the proportion of TN and non-micronodule showed a significant increase trend as ascending quartiles of PLR, LMR, and MHR (except NLR). Among men, the proportion of TN and non-micronodule showed a significant increase trend as ascending quartiles of NLR and MHR (except PLR and LMR) (Fig. 1).

Multivariate-adjusted odds ratios (ORs) of $\mathrm{TN}$ and non-micronodule with the highest quartile of the four inflammatory markers are shown in Fig. 2. MHR and PLR in women, and MHR and NLR in men had significant ORs for TN and non-micronodule. In women, the ORs in the fourth quartile of PLR for TN and nonmicronodule were 1.124(95\%C.I. 1.059-1.192) and 1.214(95\%C.I. 1.113-1.324); the ORs of MHR for TN and non-micronodule were 1.104(95\%C.I. 1.038-1.173) and 1.232(95\%C.I. 1.128-1.346); and all $P<0.001$. In men, the ORs in the fourth quartile of NLR for TN and non-micronodule were $1.112(95 \%$ C.I. $1.058-1.169)$ and 1.236(95\%C.I. $1.130-1.351$ ); the ORs of MHR for TN and non-micronodule were $1.229(95 \%$ C.I. 1.173-1.288) and 1.470(95\%C.I. 1.351-1.599); and all $P<0.001$.

\section{Discussion}

This large-scale cross-sectional study was designed to determine the relationship between four simple inflammatory indicators and the presence and size of TN. The results of this investigation show that MHR were significantly associated with the presence and nonmicronodule of TN in both genders, but PLR and NLR were significantly associated with the presence and nonmicronodule of TN in women and men, respectively.

The TN area frequently observed condition in routine physical examination and more frequent in females than in males. In this study, the prevalence of TN was $55.1 \%$ among females and $44 \%$ among males, which is consistent with those of many previous studies $[12,13]$. TN are closely related to components of the metabolic syndrome such as abdominal obesity, hypertension, hyperglycemia, and dyslipidemia $[14,15]$. Therefore, not surprisingly, the TN group also had higher mean values of anthropometric indicators, BP, FPG, TC, TG, and LDLc than that of non-TN group in the present study. (reviewer \#1, comment \#4) Considering the close association of obesity and metabolic abnormalities with inflammation markers [16, 17], we adjusted BMI, WC, hypertension, dyslipidemia, and diabetes during regression analysis to reduce the impact of these basic disease states on the relationship between those inflammation markers and TN.

One unanticipated finding was that the TN group had higher HDLc level and lower values of peripheral blood cells than that of non-TN group. However, when grouped by gender, some inflammatory indicators

Table 2 The values of the four inflammatory markers according to the presence and size of thyroid nodule

\begin{tabular}{|c|c|c|c|c|c|c|}
\hline \multirow[t]{2}{*}{ Variable } & \multicolumn{3}{|c|}{ The presence of TN } & \multicolumn{3}{|l|}{ The size of TN } \\
\hline & Non-TN & TN & $P$ value & Micronodule & Non-micronodule & $P$ value \\
\hline \multicolumn{7}{|l|}{ Females } \\
\hline NLR & $1.89 \pm 0.79$ & $1.87 \pm 0.79$ & 0.009 & $1.87 \pm 0.79$ & $1.87 \pm 0.78$ & 0.920 \\
\hline PLR & $112.0 \pm 36.9$ & $112.3 \pm 37.8$ & 0.434 & $112.1 \pm 37.3$ & $112.6 \pm 39.3$ & 0.369 \\
\hline LMR & $6.18 \pm 2.16$ & $6.27 \pm 2.18$ & $<0.001$ & $6.26 \pm 2.15$ & $6.31 \pm 2.27$ & 0.099 \\
\hline MHR & $5.60 \pm 2.25$ & $5.61 \pm 2.29$ & 0.688 & $5.58 \pm 2.26$ & $5.72 \pm 2.41$ & $<0.001$ \\
\hline \multicolumn{7}{|l|}{ Males } \\
\hline NLR & $1.81 \pm 0.77$ & $1.88 \pm 0.80$ & $<0.001$ & $1.86 \pm 0.77$ & $1.97 \pm 0.91$ & $<0.001$ \\
\hline PLR & $97.3 \pm 31.4$ & $97.4 \pm 33.2$ & 0.825 & $97.2 \pm 32.8$ & $98.3 \pm 34.9$ & 0.015 \\
\hline LMR & $5.73 \pm 1.96$ & $5.63 \pm 1.98$ & $<0.001$ & $5.66 \pm 1.98$ & $5.49 \pm 1.99$ & $<0.001$ \\
\hline MHR & $8.00 \pm 3.22$ & $8.11 \pm 3.24$ & $<0.001$ & $8.06 \pm 3.22$ & $8.33 \pm 3.33$ & $<0.001$ \\
\hline
\end{tabular}

$\mathrm{TN}$, thyroid nodules; non-micronodule was defined as the maximum diameter for TNs $>10 \mathrm{~mm}$; NLR Neutrophil-to-lymphocyte ratio, PLR Platelet-to-lymphocyte ratio, LMR Lymphocyte-to-monocyte, MHR Monocyte-to-HDL cholesterol ratio 

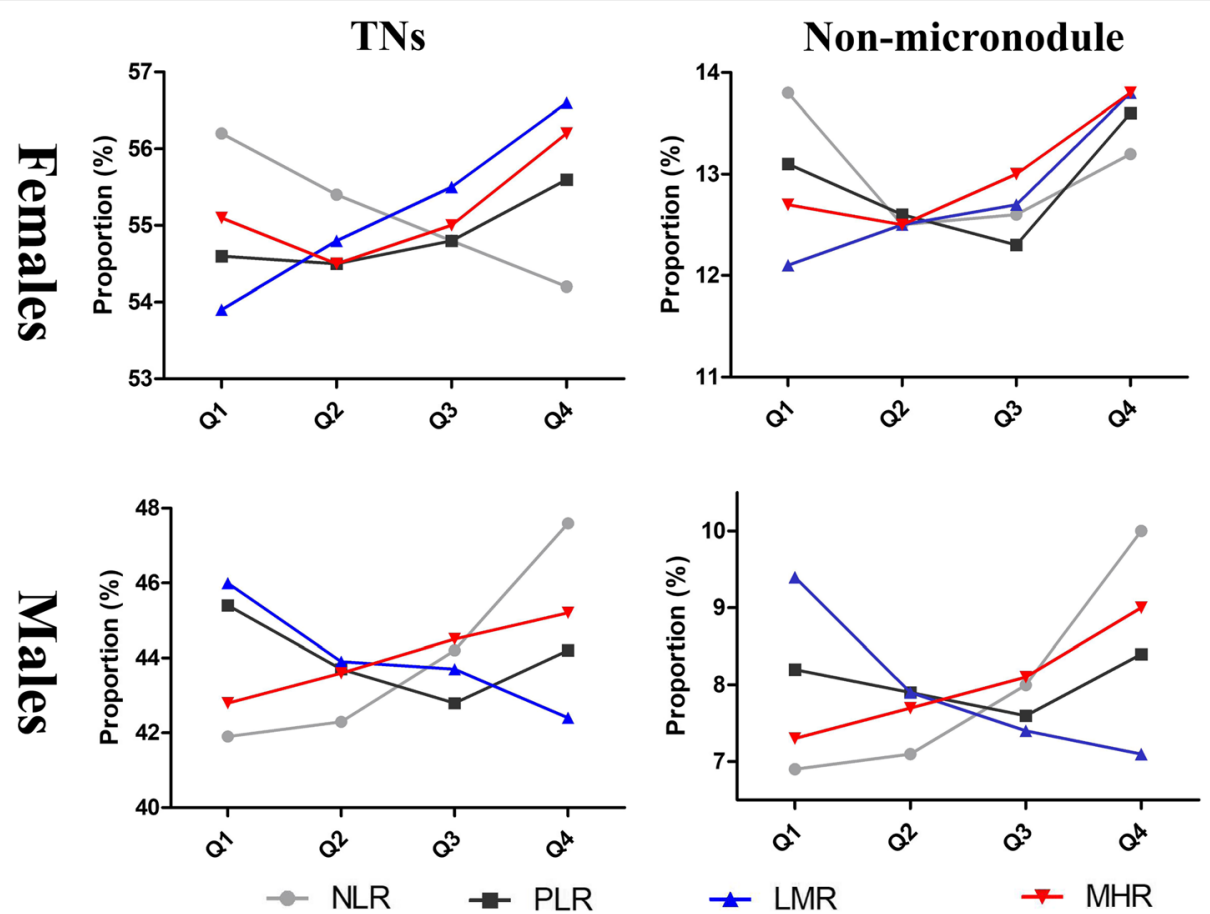

Fig. 1 The proportion of TN and non-micronodule by quartiles of the four inflammatory markers in different genders. TN, thyroid nodule; nonmicronodule was defined as the maximum diameter for TNs > 10 mm; NLR, neutrophil-to-lymphocyte ratio; PLR, platelet-to-lymphocyte ratio; LMR, lymphocyte-to-monocyte; MHR, monocyte-to-HDL cholesterol ratio

composed of these indicators in the $\mathrm{TN}$ and nonmicronodule group were significantly higher than the corresponding control group. For example, except for MHR in the TN group in women, MHR were significantly higher in the TN and non-micronodule group. These results show that the combination of some simple clinical indicators can better reflect their correlation with some diseases. In addition, gender disparity may be a key factor that must be considered when exploring the correlation between these inflammatory indicators and thyroid disease. (reviewer \#1, comment \#4).

The traditional inflammatory markers (CRP and ESR) are recommended for the assessment of rheumatic diseases, infectious diseases, and several cancers in clinical

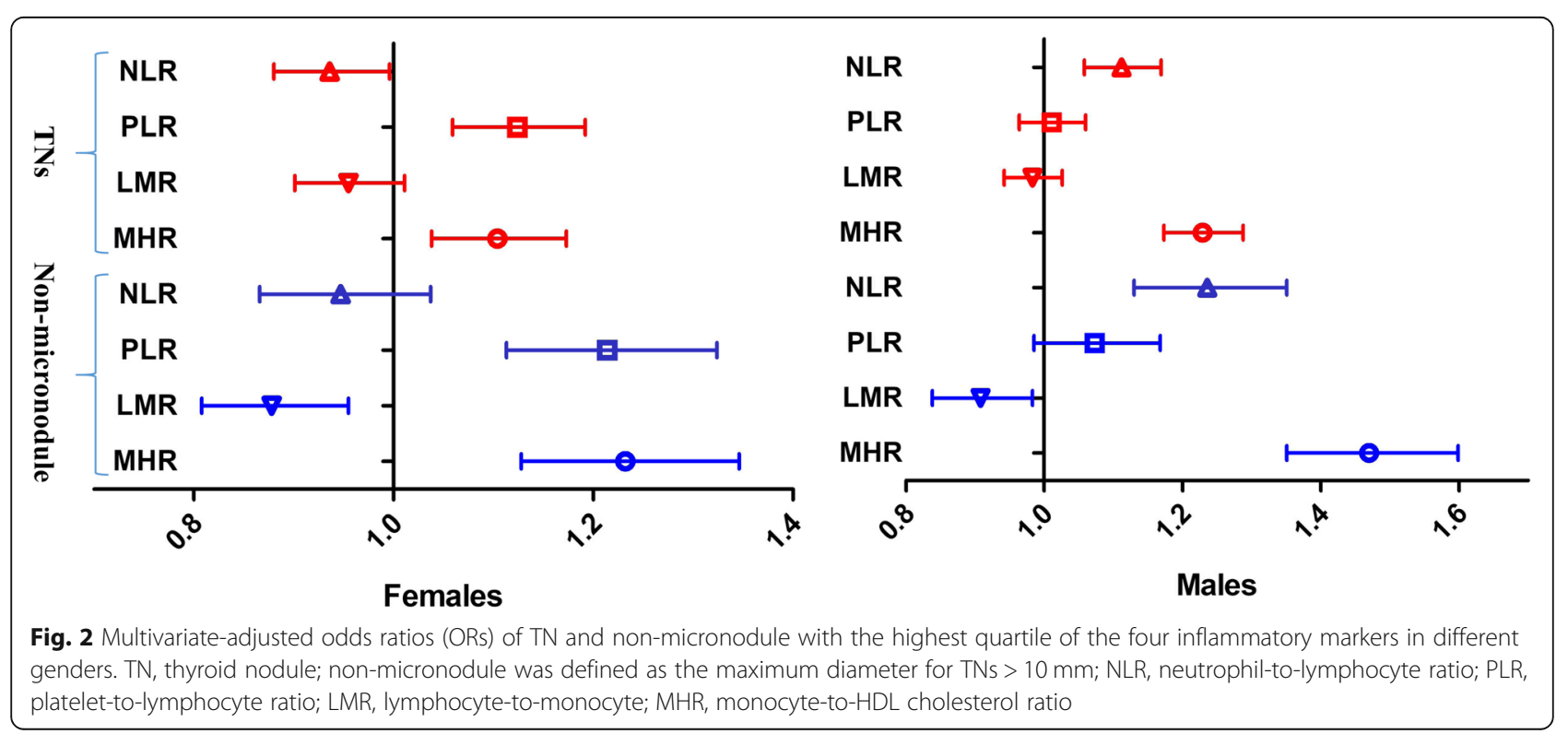


practice $[18,19]$. But the cost, difficulty in interpretating abnormal results, and possible cascade of further tests of the traditional inflammatory markers limit their clinical application. Conversely, the novel inflammatory markers (NLR, PLR, LMR, and MHR) are simply calculated from the leukocyte subsets, platelet counts, or HDLc which are routinely checked, inexpensive, readily available biomarkers. So these novel inflammatory markers have a wider range of applications, especially in primary health institutions.

In the leukocyte subsets, neutrophilia reflect a lowgrade inflammation stage and lymphopenia represent a physiological stress and poor general health $[20,21]$. So NLR reflects both inflammation and stress response which are two important immune pathways. NLR has been investigated in several studies on cancer [22], cardiovascular disease [23], and thyroid disease [24, 25]. But only one study so far had investigated the relationship between NLR and TN, which showed a negative association between high values of NLR and the presence or size of the nodules [26]. In our study, we found a positive association of NLR with the presence and size of TN in men but not in women. This gender disparity may be related to the effects of sex hormones on lymphocytes [27].

Apart from the role in blood coagulation and hemostasis, platelets also play a key role in modulating inflammatory reactions, including lymphocyte functioning. The cross-talk between blood platelets and lymphocyte have a potent effect on thyroid gland diseases [28]. So several attempts have been made to explore the connections between PLR and thyroid tumors and thyroiditis $[29,30]$. However, until now, no reported research has focused on the association between PLR and TN. Our study demonstrated that an elevated values of PLR was associated with the the presence and size of TN only in women but not in men. The effects of sex hormones on platelet and lymphocyte function may partially explain the gender differences of the relationship of PLR with TN $[27,31]$.

In the pathophysiology of tumors, lymphocytes play an important role in anti-tumor effects and lymphopenia produces an insufficient immune response against a tumor. So lower LMR was originally used to assess the prognosis of some tumors [32]. In the near future, lower LMR was also demonstrated to be associated with metabolic syndrome and vascular disease [33]. Until now, there is no research about the relationship between LMR and TN. In this study, we observed that lower LMR value was not significantly associated with TN in both men and women. The reason for the negative result is not clear but it may have something to do with the multiple roles of lymphocytes. In addition to anti-tumor effects, lymphocytes are also involved in the production of thyroid autoantibodies, which not only causes destruction but also stimulates proliferation of thyroid cells through TSH receptor activation [27, 34].

Monocytes are critical defense components a bridge between the innate and adaptive immune responses. As a source of various cytokines and molecules, monocytes are also important participants in the inflammatory pathway [35]. Contrarily, HDLc can counteract the pro-inflammatory and prooxidant effects of monocytes [36]. So the combination of monocytes and HDLc as a single ratio is promising to be a qualified indicator of oxidative stress and inflammation. Different from the above three indicators, most of the existing research focuses on the relationship between MHR and atherosclerosis [37]. So this study is also the first to explore the association between MHR and $\mathrm{TN}$, and we found MHR was significantly associated with the presence and non-micronodule of $\mathrm{TN}$ in both genders. The good performance of MHR raise intriguing questions regarding the close relationship between inflammation, metabolic disorders, and TN.

To the best of our knowledge, this might reportedly be the first large-scale study demonstrating the association between the simple inflammatory markers and the presence and size of TN. But some limitations of our study should also be mentioned. First, because of the retrospective cross-sectional design, we can not show a causal association between the four inflammatory markers and TN. Second, the study population might limit the generalisability of the results to other ethnic groups. Third, less similar research resulting in a limited possible comparisons.

\section{Conclusions}

MHR is strongly associated with the presence and size of TN irrespective of the gender. But there is a gender difference in the association of NLR and PLR with TN. These findings not only contribute in several ways to our understanding of the relationship between TN and inflammation, but also provide some candidates of simple, practical, and cost-effective inflammatory markers in the prevention and management of TN. However, further prospective and randomized studies are needed to confirme our findings.

\section{Abbreviations}

CRP: C-reactive protein; ESR: Erythrocyte sedimentation rate; LMR: Lymphocyte-to-monocyte ratio; MHR: Monocyte-to-high-density lipoprotein cholesterol ratio; NLR: Neutrophil-to-lymphocyte ratio;

PLR: Platelet-to-lymphocyte ratio

\section{Acknowledgements}

Thanks to the colleagues at the physical examination Center for accumulating a large amount of clinical data through their long and hard work. 


\section{Authors' contributions}

L-XZ and W-JM made substantial contributions to study conception and design and to the drafting and critical revision of the manuscript for important intellectual content. J-YX and Z-DB participated in the design and interpretation of the studies, and the review of the manuscript. All authors read and approved the final manuscript.

\section{Funding}

This study was supported by grants from National Natural Science Foundation of China (81671595).

\section{Availability of data and materials}

The datasets used and/or analyzed during the current study are available from the corresponding author on reasonable request.

\section{Ethics approval and consent to participate}

The research protocol was approved by the ethics committee or review committee of the Hangzhou Aeronautical Sanatorium of Chinese Air Force. Because the study was a retrospective study based on real-world situations, there was no need to obtain informed consent from the patients.

\section{Consent for publication}

All authors agree to publish this work.

\section{Competing interests}

The authors declare that they have no competing interests.

\section{Received: 28 October 2019 Accepted: 19 January 2020} Published online: 12 March 2020

\section{References}

1. Guth S, Theune U, Aberle J, Galach A, Bamberger CM. Very high prevalence of thyroid nodules detected by high frequency $(13 \mathrm{MHz})$ ultrasound examination. Eur J Clin Invest. 2009;39(8):699-706.

2. Durante C, Grani G, Lamartina L, Filetti S, Mandel SJ, Cooper DS. The diagnosis and management of thyroid nodules: a review. JAMA. 2018;319(9): 914-24

3. Kondo T, Ezzat S, Asa SL. Pathogenetic mechanisms in thyroid follicular-cell neoplasia. Nat Rev Cancer. 2006;6(4):292-306.

4. Panagiotou G, Komninou D, Anagnostis P, Linardos G, Karoglou E, Somali M, et al. Association between lifestyle and anthropometric parameters and thyroid nodule features. Endocrine. 2017;56(3):560-7.

5. Reale C, Zotti T, Scudiero I, Vito P, Stilo R. The NF-kB family of transcription factors and its role in thyroid physiology. Vitam Horm. 2018;106:195-210.

6. Haytaoglu G, Kuzu F, Arpaci D, Altas A, Can M, Barut F, et al. Correlation of vascular endothelial growth factor and vascular endothelial growth factor receptor-1 levels in serum and thyroid nodules with histopathological and radiological variables. J Lab Physicians. 2019;11(1):51-7.

7. Ammirati E, Moroni F, Norata GD, Magnoni M, Camici PG. Markers of inflammation associated with plaque progression and instability in patients with carotid atherosclerosis. Mediators Inflamm. 2015;2015:718329.

8. Watson J, de Salis I, Hamilton W, Salisbury C. 'I'm fishing really'-inflammatory marker testing in primary care: a qualitative study. Br J Gen Pract. 2016:66(644):e200-6.

9. Onoe S, Maeda A, Takayama Y, Fukami Y, Takahashi T, Uji M, et al. The prognostic impact of the lymphocyte-to-monocyte ratio in resected pancreatic head adenocarcinoma. Med Princ Pract. 2019. https://doi.org/10. 1159/000501017 [Epub ahead of print].

10. Mano Y, Shirabe K, Yamashita Y, Harimoto N, Tsujita E, Takeishi K, et al. Preoperative neutrophil-to-lymphocyte ratio is a predictor of survival after hepatectomy for hepatocellular carcinoma: a retrospective analysis. Ann Surg. 2013;258(2):301-5.

11. Inonu Koseoglu H, Pazarli AC, Kanbay A, Demir O. Monocyte count/HDL cholesterol ratio and cardiovascular disease in patients with obstructive sleep apnea syndrome: a multicenter study. Clin Appl Thromb Hemost. 2018;24(1):139-44.

12. Guo H, Sun M, He W, Chen H, Li W, Tang J, et al. The prevalence of thyroid nodules and its relationship with metabolic parameters in a Chinese community-based population aged over 40 years. Endocrine. 2014;45(2): 230-5.
13. Jiang $H$, Tian $Y$, Yan $W$, Kong $Y$, Wang $H$, Wang $A$, et al. The prevalence of thyroid nodules and an analysis of related lifestyle factors in Beijing communities. Int J Environ Res Public Health. 2016;13(4):442.

14. Chen Y, Zhu C, Chen Y, Wang N, Li Q, Han B, et al. The Association of Thyroid Nodules with metabolic status: a cross-sectional SPECT-China study. Int J Endocrinol. 2018;2018:6853617.

15. Buscemi S, Massenti FM, Vasto S, Galvano F, Buscemi C, Corleo D, et al. Association of obesity and diabetes with thyroid nodules. Endocrine. 2018 ; 60(2):339-47.

16. Serrano CV Jr, de Mattos FR, Pitta FG, Nomura CH, de Lemos J, Ramires JAF, et al. Association between neutrophil-lymphocyte and platelet-lymphocyte ratios and coronary artery calcification score among asymptomatic patients: data from a cross-sectional study. Mediators Inflamm. 2019;2019:6513847.

17. Yayla KG, Canpolat U, Yayla Ç, Akboğa MK, Akyel A, Akdi A, et al. A novel marker of impaired aortic elasticity in never treated hypertensive patients: monocyte/high-density lipoprotein cholesterol ratio. Acta Cardiol Sin. 2017; 33(1):41-9.

18. Phillips RS, Wade R, Lehrnbecher T, Stewart LA, Sutton AJ. Systematic review and meta-analysis of the value of initial biomarkers in predicting adverse outcome in febrile neutropenic episodes in children and young people with cancer. BMC Med. 2012;10:6.

19. Scott DL, Wolfe F, Huizinga TW. Rheumatoid arthritis. Lancet. 2010; 376(9746):1094-108

20. Weber C, Zernecke A, Libby P. The multifaceted contributions of leukocyte subsets to atherosclerosis: lessons from mouse models. Nat Rev Immunol. 2008:8(10):802-15

21. Azab B, Zaher M, Weiserbs KF, Torbey E, Lacossiere K, Gaddam S, et al. Usefulness of neutrophil to lymphocyte ratio in predicting short- and longterm mortality after non-ST-elevation myocardial infarction. Am J Cardiol. 2010;106(4):470-6.

22. Mattavelli D, Lombardi D, Missale F, Calza S, Battocchio S, Paderno A, et al. Prognostic nomograms in oral squamous cell carcinoma: the negative impact of low neutrophil to lymphocyte ratio. Front Oncol. 2019;9:339.

23. Prajapati JH, Sahoo S, Nikam T, Shah KH, Maheriya B, Parmar M. Association of high density lipoprotein with platelet to lymphocyte and neutrophil to lymphocyte ratios in coronary artery disease patients. J Lipids. 2014;2014: 686791

24. Ozmen S, Timur O, Calik I, Altinkaynak K, Simsek E, Gozcu H, et al. Neutrophil-lymphocyte ratio (NLR) and platelet-lymphocyte ratio (PLR) may be superior to C-reactive protein (CRP) for predicting the occurrence of differentiated thyroid cancer. Endocr Regul. 2017;51(3):131-6.

25. Celik T. Neutrophil-to-lymphocyte ratio in thyroid ophthalmopathy. Bratisl Lek Listy. 2017;118(8):495-8.

26. Erdogan Yucel E, Demiral SS. Relationship between the neutrophil to lymphocyte ratio and the presence and size of thyroid nodules. Cureus. 2019;11(1):e3866.

27. Laffont S, Blanquart E, Savignac M, Cénac C, Laverny G, Metzger D, et al. Androgen signaling negatively controls group 2 innate lymphoid cells. J Exp Med. 2017:214(6):1581-92

28. Tomczyńska M, Saluk-Bijak J. The mutual cooperation of blood platelets and lymphocytes in the development of autoimmune thyroid diseases. Acta Biochim Pol. 2018;65(1):17-24.

29. Manatakis DK, Tseleni-Balafouta S, Tzelves L, Balalis D, Tzortzopoulou A, Korkolis DP, et al. Diagnostic accuracy of preoperative neutrophil-tolymphocyte and platelet-to-lymphocyte ratios in detecting occult papillary thyroid microcarcinomas in benign multinodular Goitres. J Thyroid Res. 2018;2018:3470429.

30. Bilge M, Yesilova A, Adas M, Helvaci A. Neutrophil- and platelet- to lymphocyte ratio in patients with Euthyroid Hashimoto's thyroiditis. Exp Clin Endocrinol Diabetes. 2018. https:/doi.org/10.1055/a-0723-3441 [Epub ahead of print].

31. Khetawat G, Faraday N, Nealen ML, Vijayan KV, Bolton E, Noga SJ, et al. Human megakaryocytes and platelets contain the estrogen receptor beta and androgen receptor (AR): testosterone regulates AR expression. Blood. 2000;95(7):2289-96.

32. Ahn J, Song E, Oh HS, Song DE, Kim WG, Kim TY, et al. Low lymphocyte-tomonocyte ratios are associated with poor overall survival in anaplastic thyroid carcinoma patients. Thyroid. 2019. https://doi.org/10.1089/thy.2018. 0684 [Epub ahead of print]

33. Vahit D, Akboga MK, Samet $Y$, Hüseyin E. Assessment of monocyte to high density lipoprotein cholesterol ratio and lymphocyte-to-monocyte ratio in patients with metabolic syndrome. Biomark Med. 2017;11(7):535-40. 
34. Krátký J, Vítková H, Bartáková J, Telička Z, Antošová M, Límanová Z, et al Thyroid nodules: pathophysiological insight on oncogenesis and novel diagnostic techniques. Physiol Res. 2014;63:S263-75.

35. Sampath P, Moideen K, Ranganathan UD, Bethunaickan R. Monocyte subsets: phenotypes and function in tuberculosis infection. Front Immunol. 2018;9:1726.

36. Yvan-Charvet L, Pagler T, Gautier EL, Avagyan S, Siry RL, Han S, et al. ATPbinding cassette transporters and HDL suppress hematopoietic stem cell proliferation. Science. 2010;328(5986):1689-93.

37. Kundi H, Gok M, Kiziltunc E, Cetin M, Cicekcioglu H, Cetin ZG, et al. Relation between monocyte to high-density lipoprotein cholesterol ratio with presence and severity of isolated coronary artery Ectasia. Am J Cardiol. 2015; 116(11):1685-9.

\section{Publisher's Note}

Springer Nature remains neutral with regard to jurisdictional claims in published maps and institutional affiliations.

Ready to submit your research? Choose BMC and benefit from:

- fast, convenient online submission

- thorough peer review by experienced researchers in your field

- rapid publication on acceptance

- support for research data, including large and complex data types

- gold Open Access which fosters wider collaboration and increased citations

- maximum visibility for your research: over $100 \mathrm{M}$ website views per year

At BMC, research is always in progress.

Learn more biomedcentral.com/submissions 